\section{Remote-Sensing Applications to Support Rehabilitation of Water Infrastructure in Lapilang, Nepal}

Gl_Forum 2017, Issue 1

Page: 183 - 198

Full Paper

Corresponding Author:

barbara.riedler@sbg.ac.at

DOI: 10.1553/giscience2017_01_s183

\author{
Barbara Riedler', Magdalena Bäuerl2, Lorenz Wendt', Kerstin Kulessa ${ }^{3}$ and \\ Alexander Öze ${ }^{2}$ \\ 'Department of Geoinformatics Z_GIS, University of Salzburg, Austria \\ 2International Cooperation, Austrian Red Cross, Austria \\ 3Spatial Services GmbH, Austria
}

\begin{abstract}
Disasters such as the earthquakes that struck Nepal in spring 2015 not only cause direct impacts of death and injuries to people, but also have long-term effects such as the destruction of vital infrastructure. To support the restoration of such infrastructure, remotesensing applications can be helpful due to their potential to be complementary to in-situ data. In this study, GIS analyses are applied to Earth observation (EO) data in order to support the rehabilitation of water supply infrastructure planned by the Earthquake Recovery Operation of the Nepal Red Cross Society in the area of Lapilang, Nepal. Various aspects that are important for effective and sustainable planning are examined: (1) provision of accurate elevation information with a spatial resolution of $2 m$ to support the planning of water supply systems; (2) mapping the distribution of buildings as a proxy for population distribution in order to facilitate a spatially explicit estimate of water demand; (3) identifying potential locations for community water taps that fulfil national standards; (4) the assessment of landcover / land use in watershed areas to estimate meliorating effects and potential contamination risks. The mapped results of the analysis are not only essential for onsite field work, but can also facilitate informed decision-making processes for both short- and long-term planning of water supply systems in the area.
\end{abstract}

\title{
Keywords:
}

rehabilitation, remote sensing, water supply infrastructure, DEM generation, population mapping

\section{Introduction}

In spring 2015, two major earthquakes killed almost 9,000 and injured over 22,000 people in Nepal. 800,000 homes, livelihoods, services and infrastructure were destroyed, affecting the lives of around 8 million people. The earthquakes also severely damaged water supply schemes, including intakes, pipes and taps, and dislocated water sources (NPC, 2015). 
Subsequent to the immediate emergency response phase, the Austrian Red Cross (AutRC) in cooperation with the Swiss Red Cross and the Nepal Red Cross Society (NRCS) started an earthquake recovery operation (ERO) in 2016. The ERO comprises the four sectors of (1) shelter, (2) health and hygiene, (3) livelihood and (4) water, sanitation and hygiene (WASH) in the Dolakha district in northern Nepal. This was one of the worst affected areas, where one of the earthquake epicentres hit with a magnitude of 7.3 (OCHA, 2015).

With its WASH experience, the AutRC is supporting the rehabilitation of 18 damaged, community water supply systems in the area, where the main sources of drinking water are gravity-fed water supply systems with community tap stands, each system supplying between 15 and 150 households (ERO, 2015a).

The lack of reliable up-to-date data, such as a recent population estimate or the design and location of water supply schemes, is posing a major challenge to the rehabilitation work. In order to be able to 'build back better' and provide a well-documented basis for future interventions, the AutRC has introduced its rehabilitation work in Nepal as a case study for the research project EO4HumEn+, aiming at developing extended Earth Observation-based services and solutions for dynamic information needs in the humanitarian sector (www.zgis.at/humanitarian-services).

Using Earth Observation (EO) data and Geographical Information Systems (GIS) analysis in the humanitarian field is becoming more and more common to supplement in-situ information (Lang \& Füreder, 2015; Cowan, 2011). The approach has a variety of advantages, like area-wide coverage combined with high resolution and easily accessible data, and a huge potential to cope with the challenges that field work can face, such as inaccessibility due to remote location or dangerous situations (Lang et al., 2015; Lang and Füreder, 2015).

This study aims to show the potential of using different EO data and derived products combined with GIS analysis in the field for rehabilitating water infrastructure. The following requirements to support this work were addressed:

(1) Very high resolution digital elevation model and longitudinal cuts to support the planning of water pipelines

(2) Post-earthquake situation of the distribution of buildings as a proxy for the population distribution, resulting in an aggregated population estimate to facilitate a spatially explicit estimate of water demand

(3) Identification of potential water distribution points depending on the population density, in accordance with national standards for the location of community water taps

(4) Landcover / land use in source protection areas to estimate meliorating effects and potential contamination risks.

Although the main aim is to support the rehabilitation of water infrastructure, several aspects are considered - from the generation of a digital elevation model (DEM) to population estimation and land use / landcover. This also resembles very closely the objectives of the project EO4HumEn+, as the humanitarian application fields of population and 
environmental resources, covering water and land use /landcover, are addressed. Thus, the recent study is a good example of how the integration of various well-established methods in the domain of remote sensing and GIS analyses can give a holistic overview of a situation. Applied together they can have great explanatory potential and can function as a decisionsupport tool, in this case for the rehabilitation work of water infrastructure in an area strongly affected by a disaster.

\section{Methods}

\section{Study site}

The study site of the Village Development Committee (VDC) of Lapilang is situated in Dolakha district, around $70 \mathrm{~km}$ east of Kathmandu (Lapilang, Nepal; UL: N 27 $45^{\prime} 47^{\prime}$ ' /

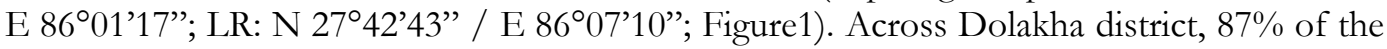
houses were registered as damaged after the earthquake in spring 2015 (GON, 2015). The ERO estimates the population of the target VDC of Lapilang to be approximately 6,200 inhabitants and 1,500 households (ERO, 2016)

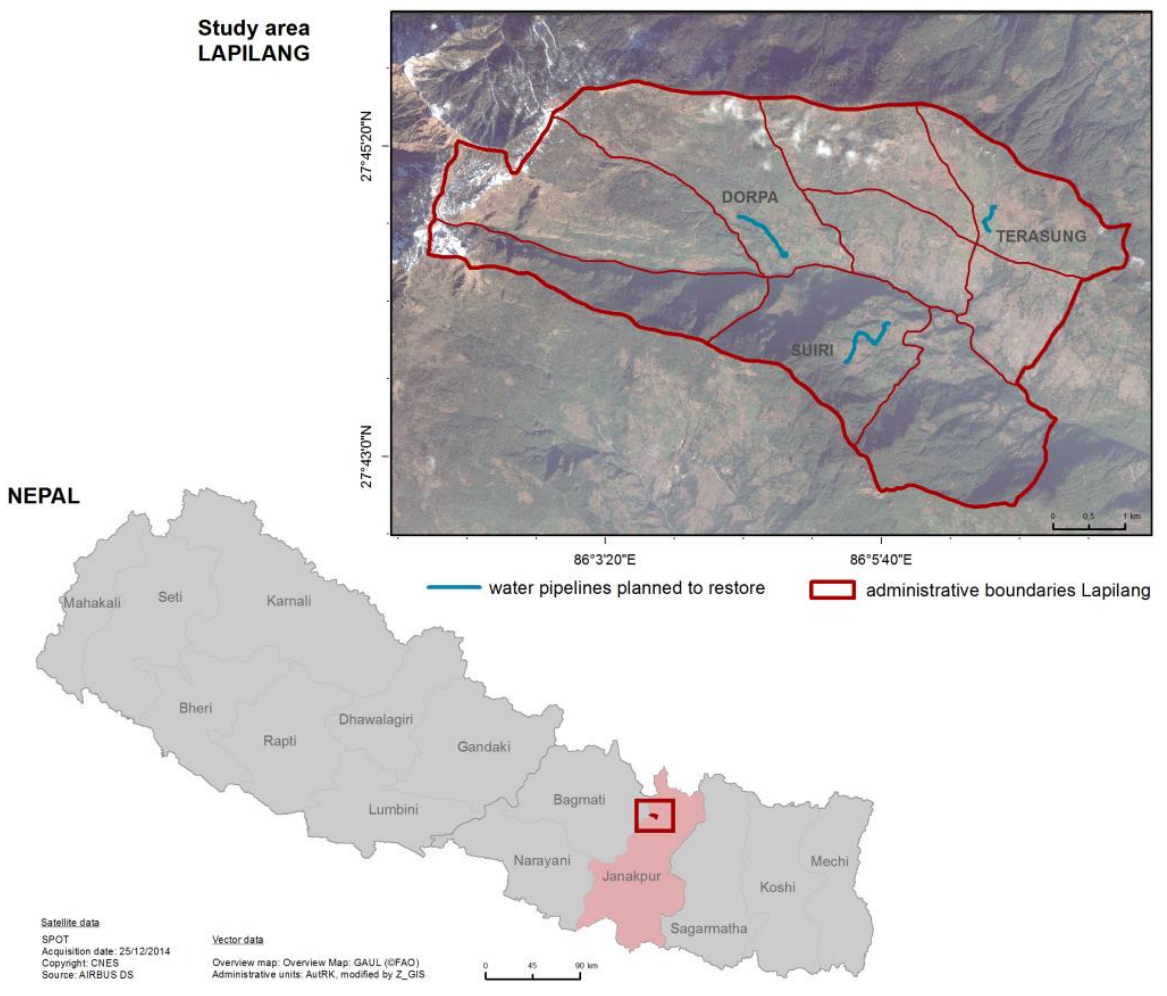

Figure 1: Study site: Lapilang, Nepal 


\section{DEM from tri-Stereo satellite imagery}

A Digital Elevation Model (DEM) with a post spacing of $2 \mathrm{~m}$ was generated using SPOT 6 satellite imagery in tri-stereo acquisition mode. With the constellation of the satellites SPOT $6 \& 7$ operating simultaneously, a daily revisit of the same location was possible (Astrium, 2013). The spatial resolution of SPOT 6 with $6.0 \mathrm{~m}$ for the multispectral and $1.5 \mathrm{~m}$ for the panchromatic image is suitable for applications in the alpine terrain. The stereo or tri-stereo acquisition mode enables the generation of DEMs using a stereo matching technique, as carried out by Eisank et al. (2015) with tri-stereo Pléiades data. Using the tri-stereo acquisition mode, three images from different angles (forward, close-to-nadir, backward) are recorded for the same location at the same time with the same sensor, as illustrated in Figure 2.

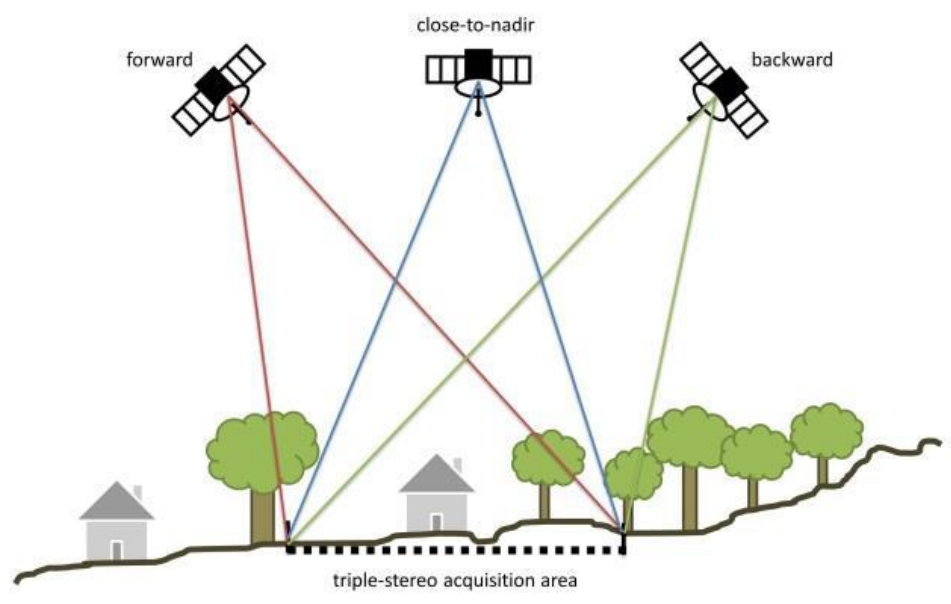

Figure 2: Tri-stereo acquisition mode

For the DEM generation for Lapilang, Nepal, the SPOT 6 primary images acquired on 25 December 2014 in tri-stereo mode containing RPC (Rational Polynomial Coefficient) files were used. The images were recorded at 04:33 UTC, which corresponds to around 10:18 NPT, before midday local time. The acquisition date and time are important considering shadows in the satellite image, which can cause errors in the DEM generation. Ideally, the acquisition time would be around noon local time to minimize shadow areas. The DEM is calculated by using the stereo matching technique in the image analysis software ERDAS Imagine ${ }^{\circledR}$. The Semi-Global Matching approach implemented in the ERDAS Imagine Photogrammetry Suite is a very robust and fast algorithm developed by Hirschmüller (2005), which combines local and global matching methods to reduce computational effort. This is a great advantage for large data sets because calculation can be done very fast. For the stereo matching, it is necessary to have a known epipolar geometry, which is ensured by the RPCfiles provided with the images. 
For the DEM calculation for Lapilang, the panchromatic images of the different acquisition angles were used because of their higher spatial resolution in comparison to the multispectral images. A block file containing all the information needed for triangulation (interior orientation, exterior orientation, sensor model and coordinate system) was calculated. After triangulation, the stereo matching was calculated pair-wise for image pairs of the tristereoscopic acquisition (forward-close-to-nadir, forward-backward, close-to-nadirbackward), resulting in a point cloud for each image pair containing one point per pixel with the elevation information. The three resulting point clouds are joined and a raster file is created out of the point cloud data.

Due to the topography of the study area (and the characteristics of the camera system), there were some large shadowed areas in the satellite imagery with very low texture. For these areas, elevation values were not calculated by the stereo matching algorithm. While small data gaps were filled by interpolation, this was not possible for the larger data gaps caused by shadows. These gaps were filled by elevation values from SRTM (Shuttle Radar Topography Mission) data. For the adjustment of the elevation of the two datasets and for the final quality control of the generated DEM, additional ground control points (GCPs) were measured in the field.

In the course of planning and designing the rehabilitation work, the project team collected GPS coordinates of the water systems from source(s) to distribution points, also marking points of interest such as landslide areas or river crossings, as a basis for longitudinal cuts. The GPS signal was then cleaned of outliers, and the vertical ( $\mathrm{z}$ ) coordinate was replaced by the more accurate $z$-value of the DEM at each location.

\section{Population distribution}

For calculating the population distribution, a manual extraction of buildings was conducted based on very high resolution (VHR) satellite imagery provided by Google Earth. The available VHR satellite images were from CNES/Astrium and Digital Globe, both presenting the post-disaster situation after the earthquake in April 2015. Based on this count of buildings a density map was created, using a kernel density estimation implemented in ESRI ArcGIS 10.4 with a search radius of $200 \mathrm{~m}$.

Additionally, the number of buildings was aggregated to the nine administrative units (wards) in the region, provided by the NRCSs. The total number of people living in the study area was provided by the ERO, which conducted a rapid water resources and population mapping in February 2016. The results of the ERO's mapping exercise cross-referenced with the number of buildings counted on the VHR satellite image served as the basis for calculating the average household size per ward.

\section{Identification of possible water distribution points}

To identify possible water distribution points, two important factors were considered: (1) horizontal distance and (2) vertical distance. Whilst the National Urban Water Supply and Sanitation Policy (MPPW, 2009) defines accessibility to a basic water supply as being within a maximum walking distance of $100 \mathrm{~m}$, the NRCS WASH technical standards recommend a 
community water tap to be accessible within a maximum horizontal radius of $150 \mathrm{~m}$ and a maximum vertical radius of $50 \mathrm{~m}$. Exceptional cases allow these standards to be widened to a maximum of $250 \mathrm{~m}$ and $80 \mathrm{~m}$ respectively (ERO, 2015b).

These search criteria were implemented in ArcGIS 10.4. The population density map which had been calculated beforehand and contour lines with an interval of $10 \mathrm{~m}$ based on the generated DEM allowed for a visual interpretation of possible water distribution points. After manually locating potential points, buffers with a radius of $150 \mathrm{~m}$ were created around these potential taps to visualize the number of buildings that could be served within the minimum horizontal distance. Elevation values extracted from the generated DEM were used for validation of the vertical requirements. Finally, the locations of the potential water distribution points were manually optimized.

\section{Landcover / land use in source catchment areas}

Land use can have positive as well as negative effects on water quality. While forests and other vegetation cover have meliorating effects, anthropogenic use such as settlements or agriculture can be a source of water contamination. The level of protection or contamination risk of a water source provides the basis for water safety planning. Therefore, it is important to estimate the catchments of each source to be used. The initial plan was to calculate the catchment area from the DEM by selecting all pixels that drain towards the location of the sources. While computationally this is not very complicated, in this case study the drainage pattern could not be extracted from the DEM at the required level of detail. The reason for this is that creeks and small water courses are often covered by vegetation, whereas the surrounding area has been cleared for agricultural use. As a consequence, the stereo matching process partly matched the top of this vegetation rather than the surface, so that the water courses are locally higher in elevation in the DEM than in reality. Therefore, the automated drainage extraction places the drainage locally at incorrect locations. To circumvent this problem, the catchments were delineated manually, by using the DEM, the extracted drainage and the satellite images. The landcover classes (agricultural / grassland / woody vegetation / bare soil or rock / settlement) were classified manually based on VHR satellite imagery provided by Google Earth.

\section{Results and Discussion}

\section{DEM and longitudinal profile}

The generated DEM with a spatial resolution of $2 \mathrm{~m}$ is shown in Figure 3, including the greycoloured data gaps caused by shadows. 

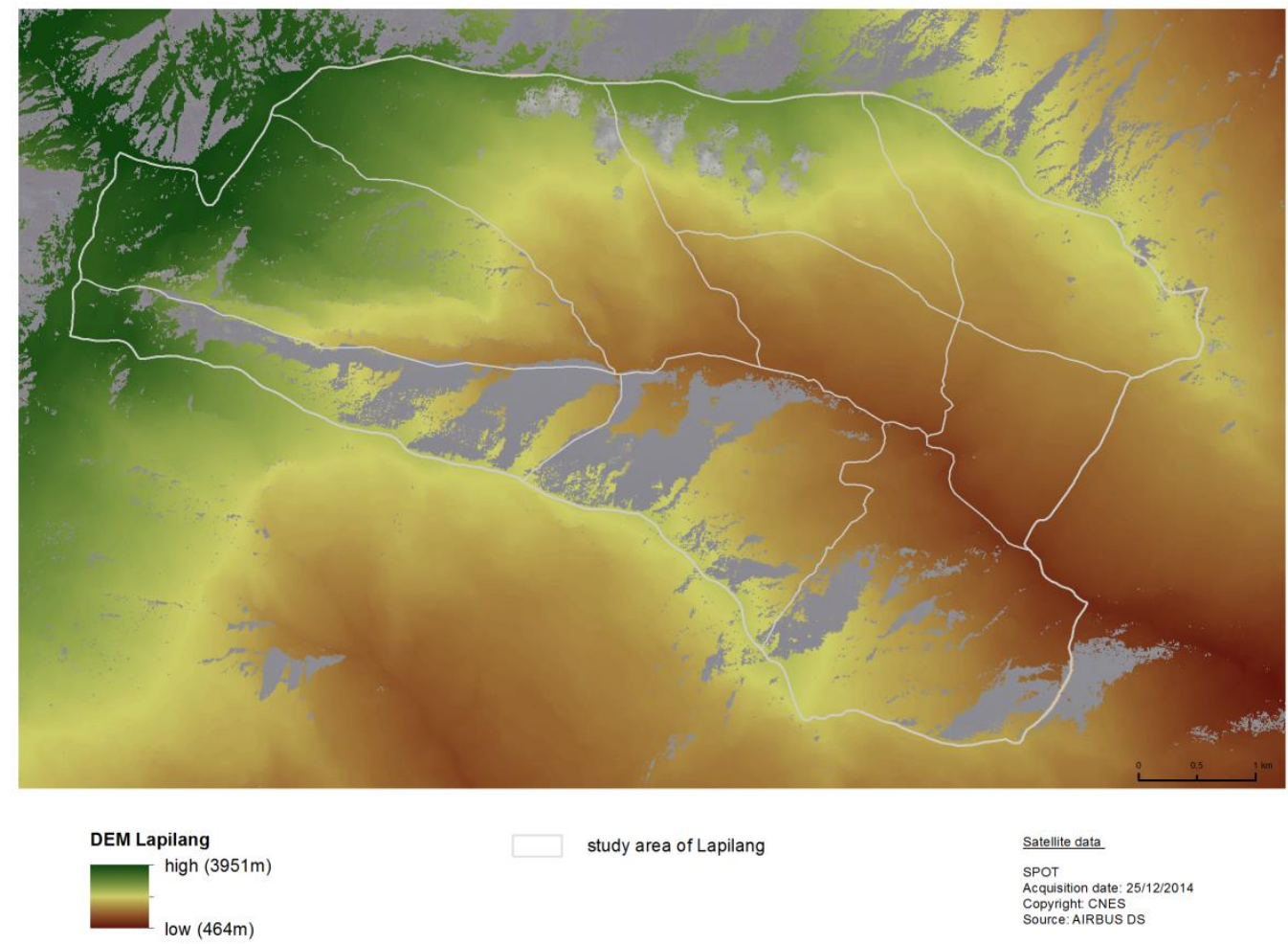

study area of Lapilang

$\underline{\text { Satellite data }}$

Acquisition date: $25 / 12 / 2014$ Copyright CNES Source: AIRBUS DS

Figure 3: Generated DEM with data gaps (in grey)

It can be seen that large areas, especially in the southern part of the study area, show such data gaps. This effect is common for optically derived DEMs, especially in alpine areas with steep terrain, as shadows depend on the topography of the study site and the position of the sun at the time of the data acquisition. The severity of this effect depends on the light conditions and the radiometric resolution of the sensor. In this study, smaller data gaps are closed by interpolation, while larger data gaps are filled with elevation information from SRTM data with a spatial resolution of around $30 \mathrm{~m}$. The location of the gaps is important when using the data for further analysis and interpreting the derived results.

Figure 4 shows the longitudinal profile of the piping system which it is planned to restore for the Suirekhola (Suiri) locality. 


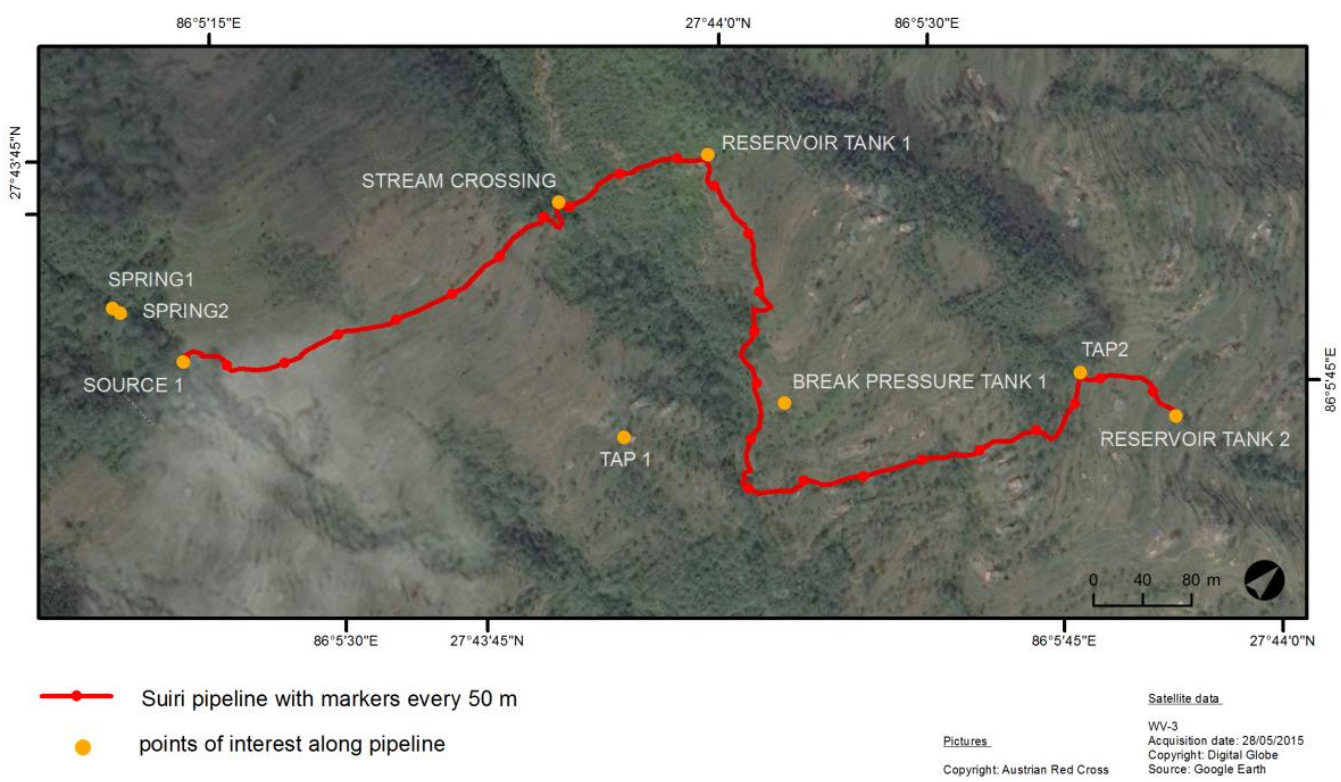

\section{Longitudinal cut along Suiri pipeline}

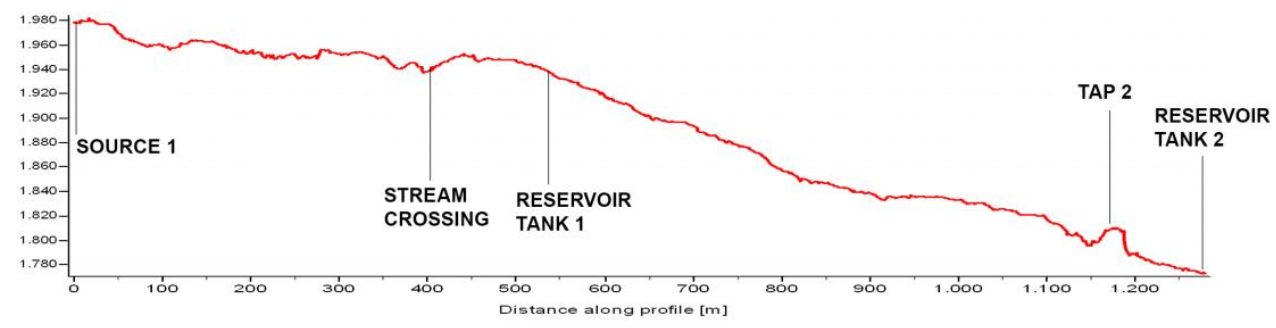

Figure 4: Longitudinal profile along the Suirekhola (Suiri) pipe system

The difference in altitude from the source to the distribution points, as shown clearly in a longitudinal cut, is relevant to support the hydraulic calculations of a gravity-fed water supply system for two main reasons. Firstly, to ensure that the system is planned and constructed in a way that water will flow freely downhill without barriers, thereby also overcoming resistance, such as wall-friction. Secondly, to ascertain that pipes are able to bear the increase of pressure that rises with altitude and might require the instalment of measures for pressure compensation. As part of a comprehensive assessment, a risk analysis for measurement errors was also conducted. Additionally, the profile can be used for the planning of river crossings and other points of particular concern along the pipeline route. 


\section{Population distribution}

In total, 1,650 buildings were extracted. As many buildings showed signs of damage, only buildings with intact rooftops were extracted and considered for further analysis. Figure 5 shows the spatial distribution of these building in the form of a density map.

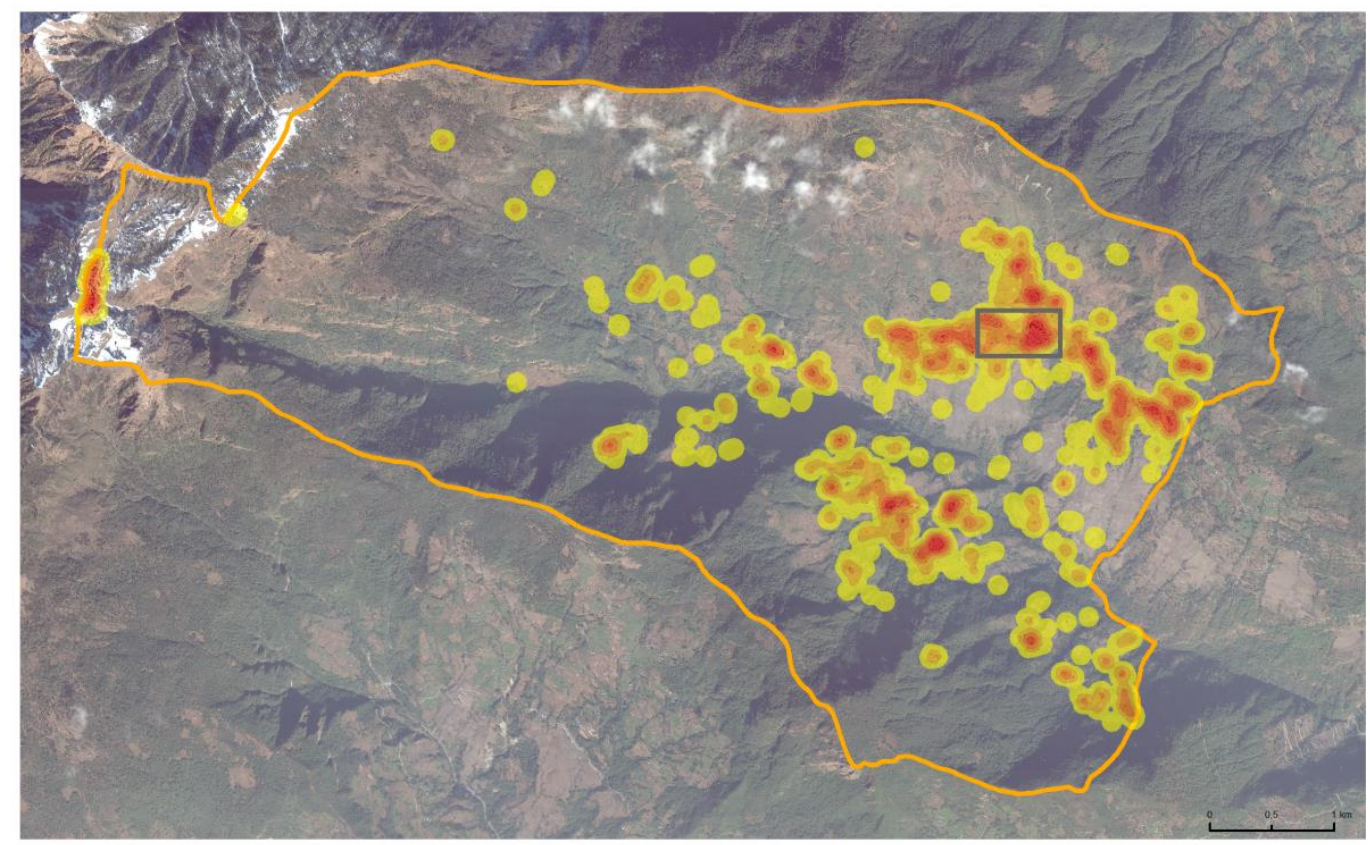

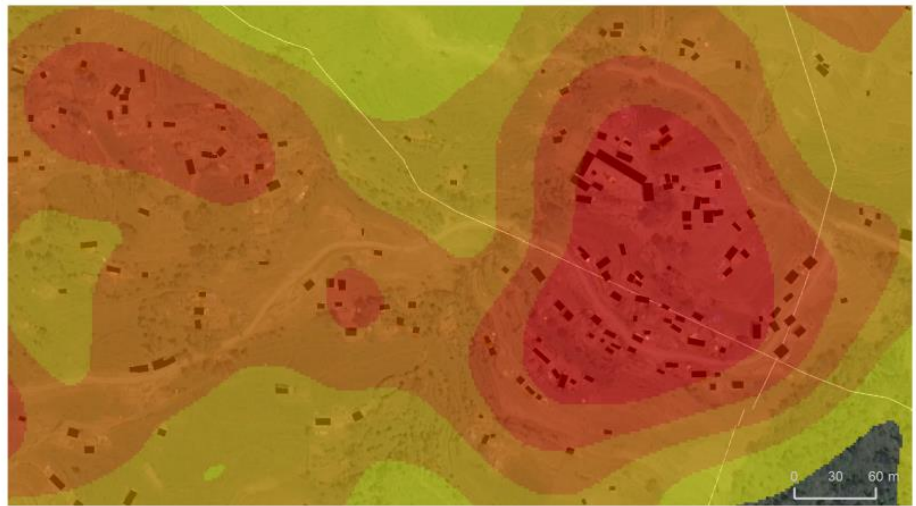

Figure 5: Population density based on single extracted buildings

\section{Population density}

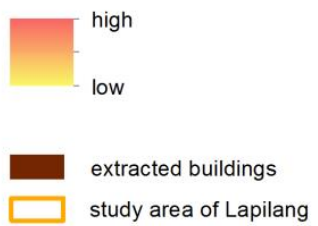

Satellite data

WV-3 SPOT Acquisition date: 28/05/2015 Acquisition date: 25/12/2014 $\begin{array}{ll}\text { Copyright. Digital Globe } & \text { Copyright CNES } \\ \text { Source: Google earth } & \text { Source:AIRBUS DS }\end{array}$

Vector data

Administrative units@AutRK, modified by Z_GIS 
Buildings occur mostly in a patchy distribution, typical for villages or farms in agricultural areas. The highest density can be found in the eastern part of the study area, with a second small density hotspot at Kuri village, in the valley, at the western border of the study site. In contrast, vast areas of the western part of the region are steep terrain, covered by dense vegetation, and thus mainly uninhabited.

A national population survey from February 2016 counted 6,234 people living in 1,474 households, resulting in an average household size of 4.3 people (ERO, 2016). In contrast, 1,650 buildings were extracted in this study, resulting in an average household size of 3.7 people. This discrepancy can be explained by the fact that a differentiation between residential houses and other buildings like stables or storage buildings was not possible from the appearance on the satellite imagery. On the other hand, the ERO survey did not provide the addresses or coordinates of the households. Therefore, the population density per ward was calculated by the aggregation of extracted buildings to the administrative ward units to support spatial decision-making processes (Figure 6). Accordingly, the absolute number of buildings and estimated people living per ward are also shown.

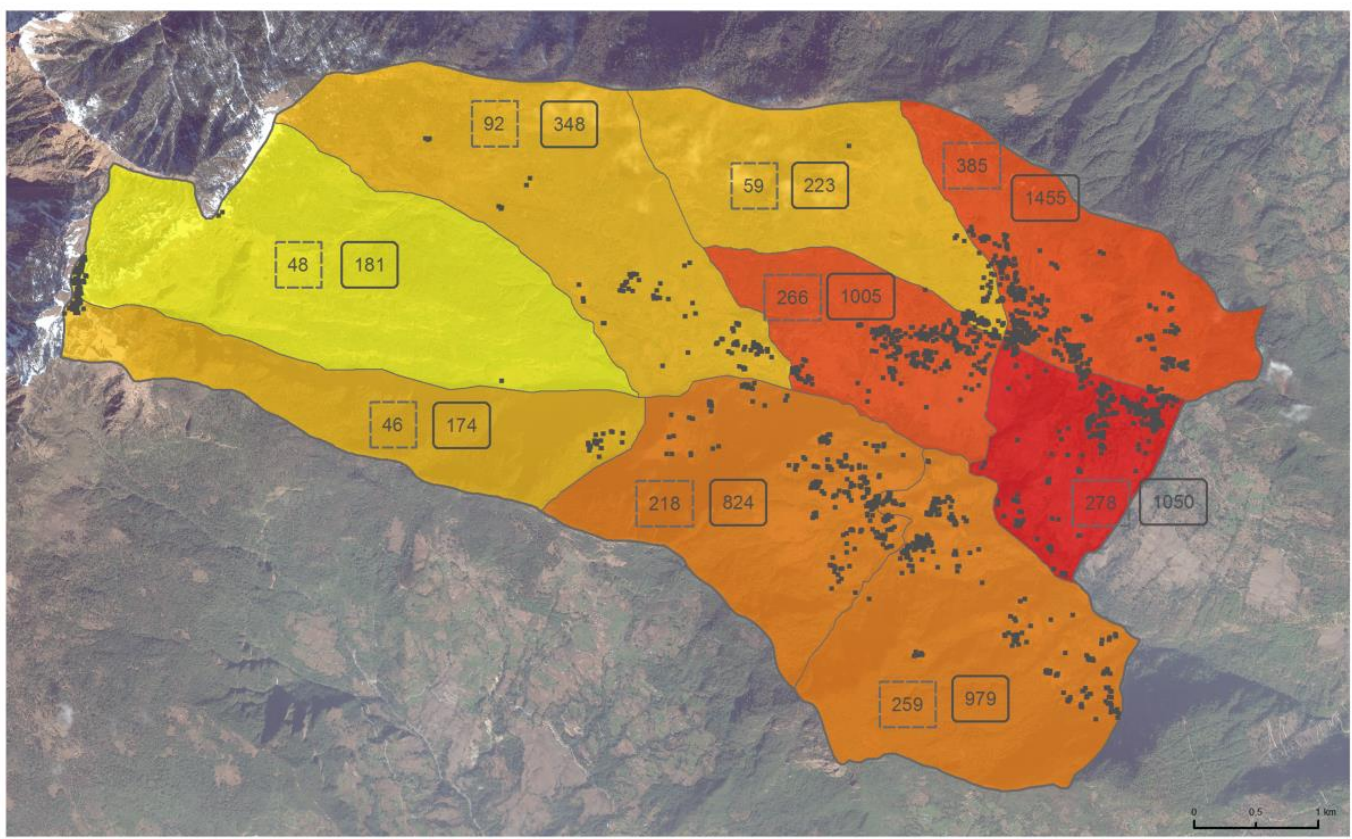

\section{Population density / ward}
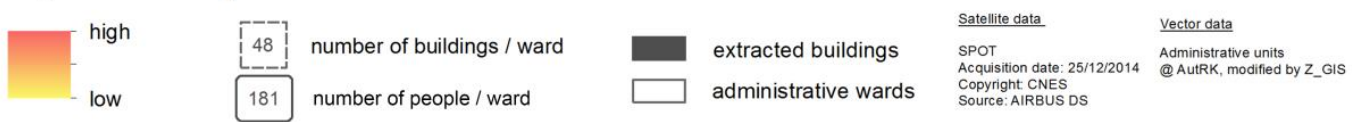

Figure 6: Population estimation per ward 
It can be seen that in the smallest ward, in the eastern part of the study area, the population density is highest. When looking at absolute numbers, most buildings and thus also most people living in the ward - assuming a uniform occupancy of buildings - can be found in the north-eastern ward. The western areas reveal the lowest building densities and absolute numbers, despite the presence of the village of Kuri at the western ward border (Figure 5).

Together, both analyses reveal an easy-to-grasp overview of where most buildings and thus also most people are located within the region of Lapilang. This might function directly as a decision-support tool in itself, allowing first conclusions about hotspot areas that might require special attention.

Additionally, these results can provide the basis for further analysis, such as the spatially explicit estimate of water demand. For the proper design of a water supply system based on water demand, a reliable population estimate and their spatial distribution are essential. However, the overall population in Nepal was last counted in the Nepal census of 2011, long before the earthquake took place. Hence, this analysis could prove essential; it can also serve to verify the results of the rapid population mapping conducted by ERO in February 2016.

Furthermore, the mapping of the buildings provides a basis for identifying the location of potential anthropogenic contamination of water sources and for determining water source protection areas.

\section{Identification of possible water distribution points}

A water supply scheme is a complex system that aims to deliver water into the direct proximity of communities, supplying as many households as possible, while being cost- and resource-efficient. For the water supply of the community of Dorpa, the restoration is planned of the main water pipeline, which was damaged during the earthquake, together with the installation of water distribution points. Decisions regarding the location of the points in relation to individual households are dependent on national and WASH technical standards of the NRCS. Based on these framework criteria, the locations of nine potential water distribution points are proposed for the community of Dorpa (Figure 7). 


\section{Population distribution}
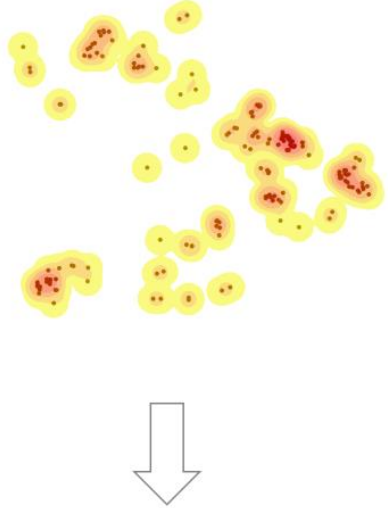

Horizontal distance
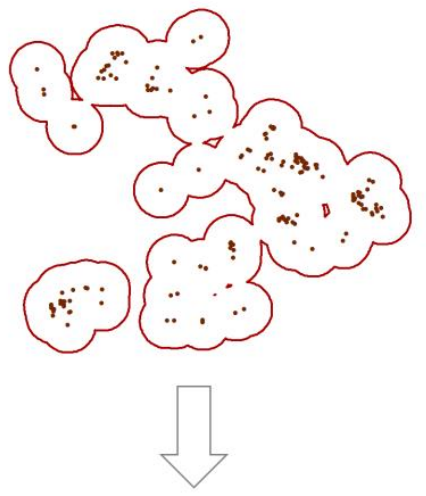

Height information
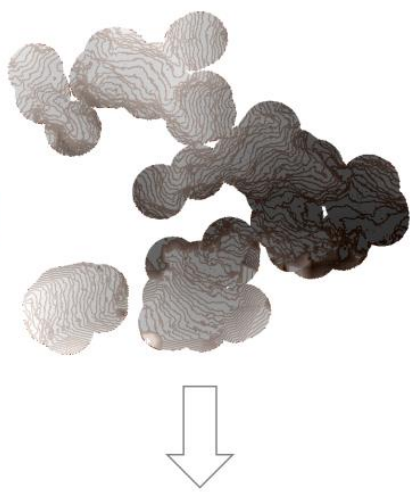

Potential water distribution points

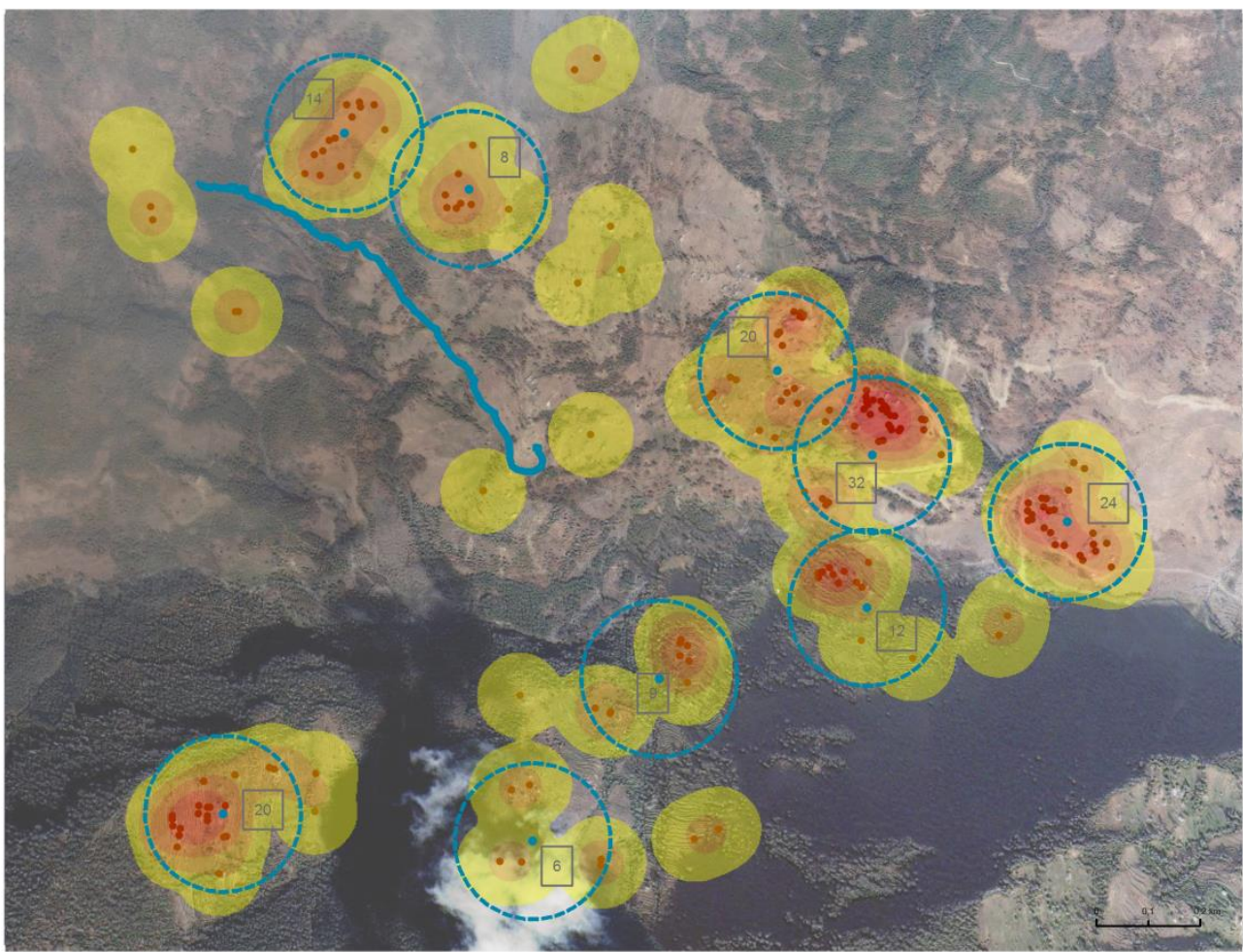

water pipeline planned to restore

- potential water distribution points

$150 \mathrm{~m}$ radius of potenital water distribution points extracted buildings

14 number of households

served within a $150 \mathrm{~m}$ radius
Satellite data

ESRI Basemap Copyright: Digital Globe, GeoEye Source: CNES Airbus

Figure 7: Potential water distribution points in the community of Dorpa 
These nine water tap stands can serve a total of 143 buildings, equating to $87 \%$ of all buildings in the community within the recommended minimum range, and leaving only a few more remotely located households to be served.

The goal for planning a sustainable water supply infrastructure is to 'leave no one behind'. However, in the northwestern part of the community, buildings are so scattered that an additional water distribution point could serve a maximum of just 3 buildings. Consequently, consideration of the remotely located houses as residential buildings should be verified in the field. While this information is provided for technical reasons, ultimately the locations and number of water distribution points to be built will be a political and economic decision.

By contrast, the highest population density is found in the eastern part of the community. In this area, all but two buildings are reached by the proposed taps, and one water distribution point can serve up to 32 buildings. The aggregated number of households will support the calculation of water demand for each potential water distribution point and will, furthermore, be decisive in determining the number of taps per distribution point. Both guiding documents (MPPW, 2009 and ERO, 2015b) agree on a minimum of 45 litres per person per day for community taps, and up to 100 litres per day or household connections. Thus, once the water supply system is calculated, the actual number of water distribution points can be determined.

\section{Landcover / land use in source catchment areas}

It is necessary to delineate the catchment area in order to assess the potential threat to the water quality of a source posed by activities and landcover upstream of the source. This can be the basis for a decision on whether a given source may be used for the supply of water for human consumption or not, even if a test of the water for pollutants or pathogenic bacteria has not shown any signs of contamination. It can also be the basis for the creation of source protection zones, in which certain potentially harmful activities such as grazing of cattle would not be allowed. Most sources to be used in this area have their catchments higher up the mountain slopes, with landcover dominated by forest. An exception is Terasung Source 1, shown in Figure 8. 

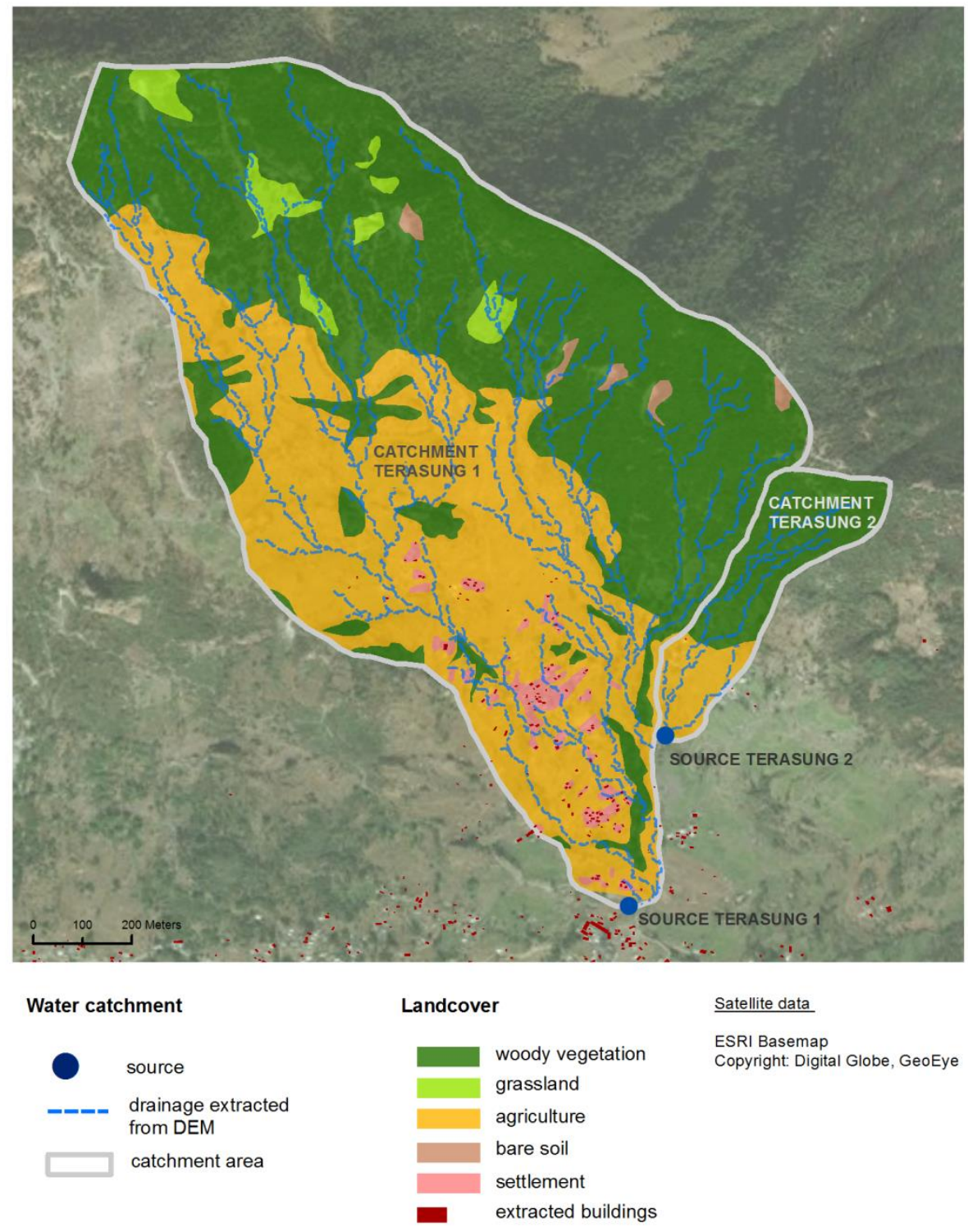

Figure 8: Delineation of the catchment area for Terasung Source 1

The catchment area of the Terasung 1 source has more than 131 ha, with a diverse landcover: $54 \%$ forest, $40 \%$ agricultural land and 3\% grassland. While settlement areas amount to just $2 \%$ of the area and have a total 130 buildings, they are located close to the water source, the nearest buildings, which are potential sources of contamination by human feces or cattle, being only $40 \mathrm{~m}$ away in an upstream direction. Additionally, the source is located directly beneath a road. Thus, it can be concluded that the source is under particular threat of contamination. 


\section{Conclusion and Outlook}

The study demonstrates the potential of remote-sensing applications to support informed decision-making and planning processes, in this case for the rehabilitation of water supply infrastructure after a disaster. While none of the methods used is particularly new, the combination of different EO data with well-established methods allows for an integrated assessment of the situation.

However, some results need careful interpretation due to methodological challenges. While the very high-resolution elevation information from satellite imagery provides a huge advantage, results from DEM generation require careful interpretation and sometimes verification in the field. This is true when, for example, the DEM of the study area shows gaps due to the mountainous topography of the site. Since verification in the field was not possible, we adopted an approach that interpolated smaller gaps and filled the larger ones with other elevation data. Because the water supply system is highly dependent on accurate elevation information as the basis for hydraulic calculations, when interpreting the results and using the data for further analysis the different resolutions at specific locations must be considered in order to avoid misinterpretation that could lead to the failure of the system.

The results of the population estimation also need careful interpretation when being used for decision making. The population estimate provided in this study is based on all extracted buildings in the area, but a differentiation between residential houses and other buildings was not straightforward from the appearance on the satellite imagery alone. Thus other buildings such as stables or storage buildings were also considered for calculating the population distribution. Additionally, an average household size was used for population estimation, assuming a uniform occupancy of buildings, independent of the size of building. Parametrization and validation with in-situ information about the type of buildings would be important to distinguish residential from other buildings and to determine indicators for the household size. With this information, a more precise population estimate regarding both population numbers and distribution becomes possible.

Perhaps the greatest potential for methodological improvement is the development of (semi)automated processes, as compared to the mainly visual interpretations conducted in this study. This would be advantageous regarding time- and thus cost-efficiency, especially when larger areas need to be covered. Applying such routines would provide more objective results, allow for easier transferability to other locations where similar information is needed, and potentially also provide results with a higher spatial accuracy. This last point is especially relevant for the identification of suitable water distribution points, where more suitable areas could potentially be identified using automated algorithms. This would in turn facilitate decisions about the location of water distribution points, because 'non-technical' aspects such as property owners need to be considered for final planning decisions.

Nevertheless, the results presented here provide valuable information for planning the rehabilitation of water infrastructure: the mapping of population distribution illustrates the overall situation of where an intact water infrastructure is essential; and highly accurate elevation information is needed for the design and construction of water supply systems, as well as for a replicable technical documentation. Moreover, the results not only support the 
planned rehabilitation of the water supply infrastructure for Lapilang, but may also be relevant for long-term planning and measurements, including the consideration of future scenarios. Based on the current population estimate and distribution, future predictions about population growth can also be made, and different scenarios, such as increased water demand due to the future installation of household connections, which is being pushed by the Nepalese Government, can be assessed more easily. The latter scenario is likely to increase water demand to 100 litres per person per day (NPPW, 2009). Furthermore, for water safety planning, the current results offer a comprehensive discussion-basis to minimize the risk of water contamination from settlements or areas used for agriculture. The delineation of a catchment and analysis of the landcover and land use in the area are necessary steps to assess the vulnerability of sources to contamination and therefore to identify possible mitigation methods, such as restricting certain activities within source protection zones, or even eliminating individual sources completely for human consumption. Finally, our approach also facilitates the taking of appropriate conservation measures, such as the protection of catchment areas or reforestation.

\section{References}

Astrium. (2013). Spot 6 \& Spot 7 Imagery User Guide. Technical Reference.

Cowan, N. M. (2011). A geospatial data management framework for humanitarian response. 8th International ISCRAM Conference Lisbon, Portugal, 1-5.

Earthquake recovery operation (ERO)(2015a). Overall Program Strategy. NRCS \& SRC Proposal Dolakha Recovery.

Earthquake recovery operation (ERO)(2015b). WASH Technical Standards. NRCS \& SRC.

Earthquake Recovery Operation (ERO) (2016). Unpublished survey on population numbers, household sizes and WASH situation in Lapilang.

Eisank, C., Rieg, L., Klug, C., Kleindienst, H. \& Sailer, R. (2015). Semi-Global Matching of Pléiades tri-stereo imagery to generate detailed digital topography for high-alpine regions. In: Car, A., Jekel, T., Strobl, J., \& Griesebner, G., (Eds.) GI_Forum_2015 - Geospatial Minds for Society, 2015, 168-177.

Hirschmüller, H. (2005). Accurate and efficient stereo processing by semi-global matching and mutual information. Computer Vision and Pattern Recognition, 2005. CVPR 2005. IEEE Computer Society Conference on. 2, 807-814.

Lang, S., Füreder, P. (2015). Earth Observation for Humanitarian Operations. 2015. In: Car, A., Jekel, T., Strobl, J., \& Griesebner, G., (Eds.) GI_Forum_2015 - Geospatial Minds for Society, 2015, 384-390.

Lang, S., Füreder, P., Kranz, O., Card, B., Roberts, S., \& Papp, A. 2015. Humanitarian emergencies: causes, traits and impacts as observed by remote sensing. In: Thenkabail,P. (Ed.), Remote Sensing Handbook (Vol. III -Water Resources, Disasters, and Urban). Taylor and Francis, New York.

Ministry of physical planning and works (MPPW). (2009). National Urban Water Supply and Sanitation Sector Policy.

National planning commission (NPC), Government of Nepal. (2015). Nepal Earthquake 2015, Post Disaster Needs Assessment.

Office for the coordination of humanitarian affairs (OCHA). (2015). Nepal: Earthquake 2015, Situation Report No. 14 (as of 13 May 2015). 